\title{
¿La escuela ha quedado obsoleta? Experiencias juveniles en el contexto escolar
}

Has the school become obsolete? Experiences youngs in the school context

\author{
Mariel Ayelen Neme ayeneme@hotmail.com \\ http://orcid.org/0000-0002-6194-182X \\ Facultad de Ciencias Económicas, Jurídicas y Sociales; \\ Universidad Nacional de San Luis (Argentina)
}

\section{Resumen}

Mientras transitamos el siglo XXI ostentando grandes avances tecnológicos y un estilo de vida caracterizado por la globalización y el consumo, la escuela ¿se ha vuelto obsoleta? El presente texto, forma parte de mi investigación de tesis de maestría que aborda a partir del análisis de las experiencias escolares de las y los jóvenes, los sentidos que se (re)construyen sobre estudio y trabajo. En este marco, este artículo propone acercar la mirada a las experiencias educativas en el nivel medio a fin de comprender los sentidos que los jóvenes (re)construyen 
sobre la escuela en tiempos en los que se discute ampliamente la razón de ser de esta institución educativa. Para lograr el objetivo se realizó desde la perspectiva cualitativa un estudio de caso que consistió en entrevistas semi-estructuradas realizadas a diez jóvenes que transitan sexto año de una escuela pública autogestionada en la ciudad de Villa Mercedes, San Luis, Argentina. Se buscó en este escrito describir algunos aspectos de las experiencias juveniles sin pretender imponer verdades ni hacer generalizaciones sino con el fin de aportar al debate y así comprender las particularidades de la relación que los jóvenes establecen con la escuela.

Palabras clave: Jóvenes; experiencia escolar; nuevas tecnologías.

\begin{abstract}
As we move through the 21st century showing great technological advances and a lifestyle characterized by globalization and consumption, has the school become obsolete? This text, is part of my research thesis of masters that addresses from the analysis of school experiences of young people, the senses there are built and rebuilt on study and work. In this framework, this article proposes to approach the educational experiences at the high school in order to understand the meanings that young people reconstruct about school in times in which the reason for being of this educational institution is widely discussed. To achieve the objective, a case study was carried out from a qualitative perspective consisting of semi-structured interviews conducted with 10 young people who are in the 6th year of a self-managed public school in the city of Villa Mercedes, San Luis. Argentina. We sought in this paper to describe some aspects of youth experiences without trying to impose truths or make generalizations but in order to contribute to the debate and thus understand the particularities of the relationship that young people establish with the school.
\end{abstract}

Keywords: Young people; school experience; new technologies.

La escuela actual es motivo de preocupación y debate no solo en el campo de la investigación sino como tema de agenda prioritario de las políticas públicas. Los índices de repitencia y deserción, sobre todo en el nivel medio, los deficientes resultados de las pruebas académicas, las desigualdades tanto sociales como culturales que produce y reproduce son indicadores de que la institución está en crisis. Tal como señalan Nuñez y Retichever (2015) la sensación de ausencia de sentido de la experiencia escolar, la nostalgia por un tiempo pasado que se 
recuerda en sus mejores trazos, la ansiedad e incluso la desazón que muchos docentes pueden sentir ante alumnos muy distintos a los que suponían que tendrían, parecen ser las coordenadas que organizan el debate contemporáneo en nuestro país. Las causas de esta sensación de crisis e incertidumbre, tal como sostiene Sibilia (2012) se deben a que la escuela es una tecnología de época, aunque su presencia se haya naturalizado y su inexistencia parezca inimaginable, su invención se dio en un espacio temporal concreto y en una situación socio histórica determinada que hoy es obsoleta.

Haciendo una pequeña génesis histórica puede afirmarse que la escuela pública se vincula a la construcción del Estado Moderno durante el siglo XIX. En Argentina la escuela primaria en sus inicios incluía a un grupo reducido de niños, sin embargo desde su conformación se manifestó su principio universalista y la obligatoriedad de escolarizar a toda la población infantil según estableció la Ley 1420 sancionada en 1884. En cuanto a los primeros establecimientos destinados a la enseñanza secundaria éstos se constituyeron bajo el gobierno de Rivadavia con la finalidad de formar a los intelectuales de la clase dirigente (Macri, 2010). De este modo el principal objetivo de la escuela moderna fue reforzar la legitimidad del poder a través de la constitución de una red escolar destinada a socializar a las nuevas generaciones para convertirlos en "buenos ciudadanos". Así, la formación del Estado Nacional estuvo muy ligada a la constitución de los Sistemas Educativos Nacionales. Por lo tanto, el Estado y la constitución de las fronteras nacionales, una determinada definición del conocimiento y el saber, la conformación de una sociedad meritocrática, la consolidación de la familia nuclear, formaron una red que según Tiramonti (2011) reguló el mundo moderno.

Esta situación cambió en la actualidad como resultado de una profunda transformación cultural apoyada principalmente en las tecnologías electrónicas y digitales, regida por los excesos de producción, consumo y la interconexión en tiempo real por medio de redes globales de comunicación. Sin embargo la escuela permanece allí, con una gramática que parece inmutable. Evidentemente hay una divergencia de época que no puede soslayarse ni tampoco ha podido acortarse ya que aunque se han modificado algunos aspectos, los cambios aún no logran ser estructurales. En este escenario los y las jóvenes que transitan la escuela se enfrentan cotidianamente a esta dicotomía entre una conjugación informática digital e interactiva por un lado y por el otro una escuela que sigue operando con la tiza y el pizarrón, horarios fijos, prueba escrita y lección. ¿Qué sentidos encuentran los y las jóvenes a su recorrido por la escuela? ¿Qué les gusta y que cambiarían? ¿Cómo son sus experiencias escolares con las nuevas tecnologías? ¿Prefieren la enseñanza escolar presencial o preferirían que la escuela fuese virtual? Las respuestas a estos interrogantes desde las experiencias juveniles pretenden aportar al debate sobre la escuela y las relaciones con las y los jóvenes. 


\section{Sobre jóvenes y experiencias}

Es preciso conceptualizar en primer lugar desde que lugar se habla cuando se habla de los jóvenes. La emergencia de las juventudes como sector social diferenciado es bastante reciente en perspectiva histórica. Varios autores ( Feixa,1999; Chaves, 2005; Bergé, 2007) coinciden en que fue luego de la Segunda Guerra Mundial, teniendo como eje Europa y Estados Unidos, cuando ciertas condiciones sociales que se dieron en ese período permitieron su emergencia, entre ellas: la consolidación del Estado de Bienestar que creó las condiciones para un crecimiento económico sostenido y para la protección social de los grupos dependientes, permitiendo la ampliación de las esferas de la educación y el ocio en determinados sectores; la crisis de la autoridad patriarcal que llevó a nuevas esferas de libertad juvenil; el nacimiento de un mercado destinado específicamente al consumo de los jóvenes; la ampliación de los medios masivos de comunicación y su papel en la identificación de una dimensión generacional e internacional de "ser joven", cambios en la esfera de la educación instaurando la "educación secundaria para todos" ampliando las posibilidades de acceso a la educación superior; el arribo de toda una serie de estilos distintivos de ropa y música consolidando sin lugar a dudas a la generación más joven.

Uno de los investigadores pioneros que sembró las bases para estudiar a la juventud desde la cultura fue Pierre Bourdieu (1990) quien en uno de sus escritos" la juventud no es más que una palabra" instaló la idea de la categoría juventud como construcción, como representación ideológica de la división de los grupos. En nuestro país Mario Margullis (1996) es el pionero en la temática y con su obra titulada "La juventud es más que una palabra" hizo hincapié que el término tiene usos particulares sentidos singulares y efectos concretos. Ambos investigadores uno en Europa otro en Latinoamérica, instauraron la idea de pensar la juventud en contexto de modo que hoy el término juventud es utilizado como una categoría analítica construida en el juego de relaciones sociales (Chaves, 2010). Esta noción implica situar a los jóvenes en un contexto social, económico y cultural determinado. En esta misma línea, Feixa explica que para que exista la juventud deben existir, por una parte, una serie de condiciones sociales (normas, comportamientos e instituciones que distingan a los jóvenes de otros grupos de edad) y, por otra parte, una serie de imágenes culturales (valores, atributos y ritos asociados específicamente a los jóvenes). Tanto unas como otras dependen de la estructura social en su conjunto, es decir, de las formas de subsistencia, las instituciones políticas y las cosmovisiones ideológicas que predominan en cada tipo de sociedad (Feixa, 1999). 
Por otro lado, se consideró para el análisis la categoría "experiencia" como un buen camino de acceso a los sentidos que los jóvenes otorgan a la escuela ya que, tal como plantea Larrosa (1996 : 18) la experiencia es aquello que nos pasa: "No lo que pasa, sino lo que nos pasa (...) lo que nos afecta en lo propio". Larrosa retoma de Benjamín la idea de que la experiencia es aquella que nos atraviesa y deja huella, nos con-mueve; diferenciando entre "lo que pasa" y lo que "nos pasa", es decir entre lo que podemos conocer y a lo que podemos atribuir un sentido en relación a nosotros mismos. La experiencia sería, entonces el modo en que atribuimos sentido, en este caso la cultura juega un papel central de mediación.

En otras palabras, enfocando la mirada en los sentidos que los jóvenes construyen alrededor de su experiencia escolar, puede desentramarse el valor simbólico y la apropiación que ellos otorgan a lo que la sociedad les pone a disposición.

Como afirma Duschatzky (1999), no se trata de descubrir la esencia de la escuela inscripta en su naturaleza, sea ella la de educar para la integración social o para la reproducción de las desigualdades, se trata de interpretar los deslizamientos de sentido inesperados que aparecen.

\section{Metodología}

Desde una perspectiva cualitativa, el trabajo de campo consistió en un corpus de diez entrevistas no estructuradas a jóvenes alumnos de una escuela pública de la ciudad de Villa Mercedes, San Luis, Argentina. La selección de los casos fue intencional, de acuerdo a las disponibilidades de la dinámica escolar pero con el único requisito que se encontraran cursando o rindiendo materias del último año y que fuesen alumnos de la escuela. El esquema de entrevista se diseñó con preguntas abiertas que permitieran comprender los sentidos de los jóvenes en relación a la experiencia escolar. La cantidad de entrevistas se determinó en términos de Glasser y Strauss (1967) por saturación teórica cuando ya no emergieron datos nuevos. El análisis de datos se realizó con el software Atlas Ti. Este programa informático permitió codificar las entrevistas, obtener un listado de códigos descriptivos y establecer las conexiones entre ellos mediante la elaboración de redes semánticas que fueron incorporadas a modo de gráficos en este artículo.

\section{El contexto institucional}


La institución escolar de nivel secundario en la que se realizó el estudio de caso es una escuela pública autogestionada. Esta modalidad de gestión es única en el país y nació en la provincia de San Luis, Argentina, a los fines de la década del noventa con la intención de reconfigurar un sistema educativo descentralizado del Estado. El modelo está basado en las "escuelas charter" que se desarrollaron en la década del ochenta en Estados Unidos. Son escuelas públicas independientes fundadas por docentes, padres, $u$ organizaciones sin fines de lucro. Siguiendo a Cicioni (1998), este tipo de escuelas están liberadas del micro management burocrático y regulatorio para organizarse alrededor de una misión principal, de una currícula, de métodos de enseñanza, y de formas organizativas alternativas, de acuerdo a sus propias preferencias. Son escuelas públicas porque son gratuitas y de acceso universal. Reciben fondos del Estado de acuerdo a la cantidad de alumnos que asisten y gestionan por sí mismas la totalidad de su presupuesto. Esta propuesta en un comienzo encontró en la provincia de San Luis serias resistencias en la comunidad educativa, especialmente en la Asociación de Magisterio Puntano, en la Coordinadora de Padres de San Luis, en la llamada asociación de "padres auto convocados" de la ciudad de Villa Mercedes, quienes realizaron movilizaciones, marchas, cartas y denuncias en los medios aduciendo a que este tipo de escuelas tendía a la privatización de la educación. El fundamento de esta aseveración era que la supuesta autonomía que promulgaba este tipo de escuelas estaba concentrada en manos de asociaciones educacionales conformadas por un número reducido de personas. Además se consideraba que los docentes que eran incluidos en estos establecimientos educativos quedaban ante una situación de vulnerabilidad ya que en la mayoría de los casos eran contratados temporalmente por las asociaciones educacionales sin tener las garantías laborales que ofrece el Estado. Sin embargo más allá de los reclamos el gobierno provincial realizó de todos modos la convocatoria nacional a aquellas asociaciones interesadas en formar parte de este tipo de instituciones.

En este contexto, la escuela pública auto gestionada Nro. 8 "Leonardo Da Vinci" -tras haber sido seleccionada en esa convocatoria- inició sus actividades en el mes de febrero de 2001. La institución funcionó desde su nacimiento en distintos edificios hasta 2015, cuando se le otorgó edificio propio en un barrio periférico aproximadamente a 4 kilómetros del centro de la ciudad de Villa Mercedes. La escuela está integrada por treinta docentes y diez no docentes (administrativos y ordenanzas) y aproximadamente mil alumnos que concurren entre los dos turnos (mañana y tarde).

A partir de las observaciones de la dinámica escolar y de las entrevistas realizadas al personal de la institución pudieron conocerse aquellas reglas no escritas que definen los límites entre el adentro y el afuera, determina lo prohibido y lo admitido y los sentidos que los actores le 
asignan a la escuela, lo cual constituye la identidad institucional una definición consensuada de lo que el establecimiento es, según las recurrencias de acción que conforman su estilo. El perfil institucional de la escuela, la propuesta pedagógica y la existencia de espacios de participación de los estudiantes son indicadores del tipo de construcción colectiva que se lleva a cabo.

Un primer sentido particular de la escuela lo constituye la enseñanza personalizada. La directora relacionó esta enseñanza personalizada con el proyecto intra institucional de tutorías y con la búsqueda de la escuela a obtener una calidad educativa.

Una segunda característica identificada por los miembros de la escuela es la libertad, característica que apareció asociada a la auto-regulación ya que no existen reglas estrictas ni amonestaciones que sancionen conductas indeseadas, tampoco se exige uniforme. Esta libertad según percepciones del personal de la escuela, trae consigo la tranquilidad en los alumnos. Otra característica que fue mencionada por el personal de la institución es la motivación a los jóvenes por parte de los profesores y de la directora. Esta motivación los adultos de la escuela la relacionan con el vínculo que construyen con los alumnos. Un vínculo de cordialidad, diálogo y apoyo para realizar actividades relacionadas con su progreso, con la autoconfianza, la perseverancia y el optimismo.

Estas características más allá de la modalidad de la institución, relacionadas con el clima y la relación que ésta propicia y las percepciones de sus miembros sobre ella, otorgaron al análisis características particulares sobre los sentidos que adquiere para los jóvenes esta escuela en particular y el modo en el que problematizan la (in)compatibilidad entre la gramática escolar y las nuevas tecnologías.

\section{Sentidos juveniles en sus recorridos por la escuela}

A través de los discursos los jóvenes dieron cuenta de aquello que perciben de su paso por la escuela. Como se expresó anteriormente históricamente la escuela ha sido la institución legitimadora de saberes reproduciendo aquellos que eran considerados valiosos para la sociedad, nadie ponía en duda que aquello que se valoraba como conocimiento se aprendía en la escuela. Hoy, en medio de los cambios y movimientos, esto parece estar en crisis. Y los jóvenes lo expresan en sus discursos, sus respuestas se encuentran reflejadas en la siguiente figura: 


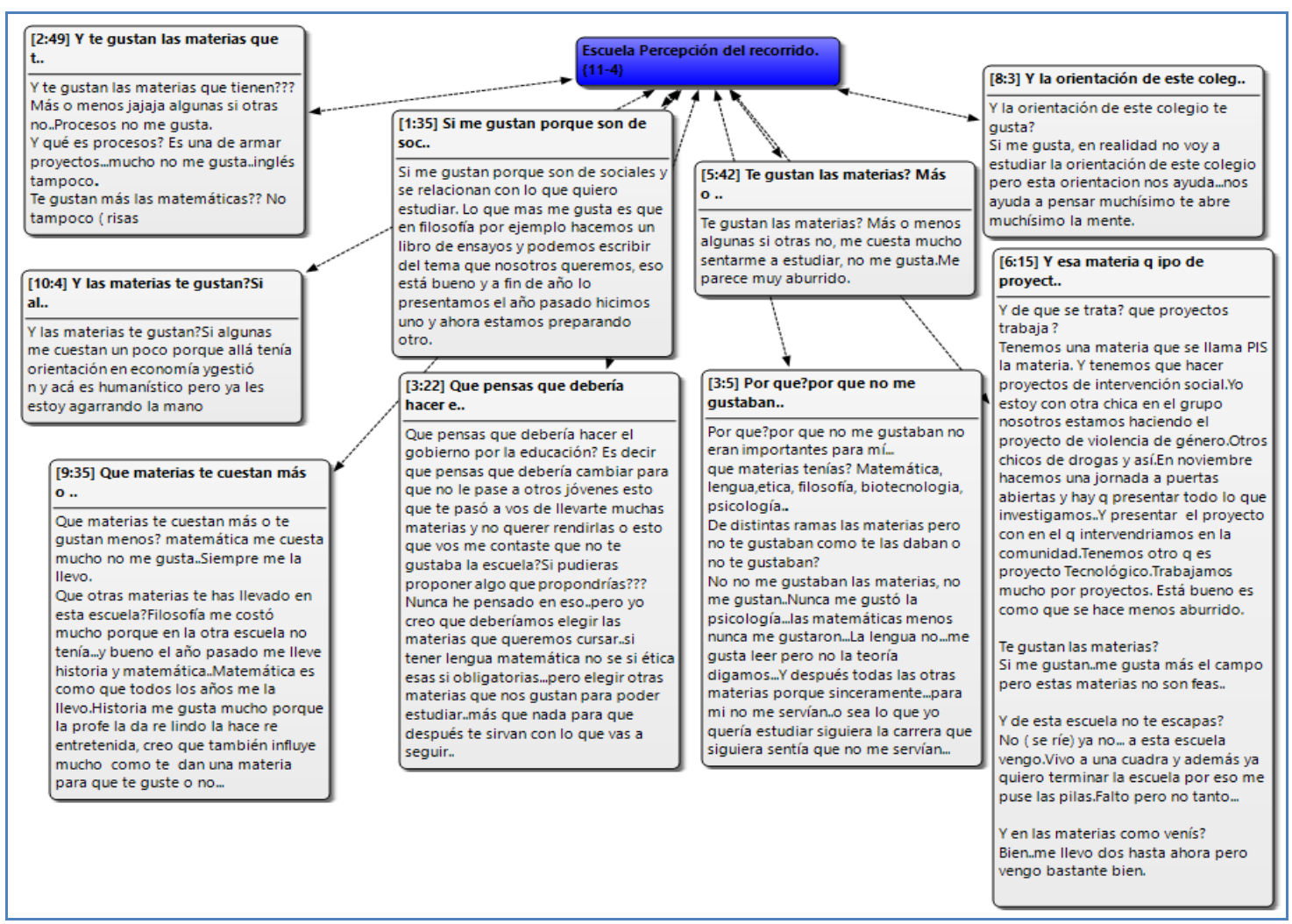

Figura 1. Percepción del recorrido

Como se muestra en la figura precedente, los jóvenes expresan las materias que les gustan y las que no, que algunas les cuestan más que otra, pero lo recurrente en sus respuestas fue que en varias oportunidades utilizaron el adjetivo "aburrido" para referirse al estudio en el contexto escolar:

Me cuesta mucho sentarme a estudiar, no me gusta, me parece muy aburrido ( $M$, sexo masculino, 20 años).

Trabajamos por proyectos, está bueno es como que se hace menos aburrido (B, sexo femenino, 18 años).

Allí el calificativo da cuenta de su percepción sobre la escuela como aburrida, y ante esta modalidad de trabajos (por proyectos) se hace "menos aburrida" lo cual no significa que considere a la escuela divertida o interesante.

Otra de las jóvenes entrevistadas, ya había terminado de cursar el secundario pero le habían quedado materias pendientes que luego de dos años había vuelto a rendirlas para tener su título secundario. Esta joven manifestó que: 
No me gustaba asistir porque las materias no me interesaban, ninguna me importaba porque no creía que fueran a servirme para el futuro, no por la orientación de la escuela sino en general porque la educación en las escuelas no es interesante ( $T$, sexo femenino, 20 años).

Nuevamente el "no me interesaban" y "no creía que fueran a servirme para el futuro" se vincula con la valoración de la escuela como aburrida y se agrega otro valor relacionado al aburrimiento que puede ser tomado como una de las causas del mismo: la utilidad. "Es aburrida porque es poco útil".

El "aburrimiento" apareció en las entrevistas como una percepción de su recorrido escolar vinculado al interés. Usan este término para calificar una escuela que no los estimula, les genera cansancio, distracción y molestia.

Por un lado, esta valoración puede analizarse desde el contexto de incertidumbre ante la crisis de las instituciones que anclaron los sentidos de la vida durante la Modernidad. La escuela no ha podido adaptarse a la nueva condición de globalización que tiene el orden mundial. Tal como afirma Tiramonti (2011) todo ha cambiado menos la escuela. Una condición de cambios y desarticulación, al decir de Bauman (1999), una modernidad líquida que se presenta como ruptura a las sólidas verdades. La escuela actual según Corea y Lewcowicz (2004) funciona como "galpón" en el sentido que en ella existe una aglomeración de personas que comparten un espacio pero ninguno está en la misma situación que el otro. En esas condiciones, los vínculos cambian de cualidad, están sometidos a los encuentros y desencuentros. En este sentido el "aburrimiento" podría interpretarse como la manifestación de un desfasaje de época. Por otro lado, un segundo modo de abordar el análisis del "aburrimiento" seria preguntarse ¿por qué los jóvenes reclaman que las clases de la escuela sean divertidas o entretenidas si la función de la escuela es enseñar?

Puede afirmarse como posible respuesta que, en esta época de constantes estímulos provenientes del entorno, la motivación juega un papel muy importante en las percepciones juveniles y el aburrimiento se presenta como un síntoma de los límites de la experiencia pedagógica para motivar a los alumnos.

En este contexto la escuela ensaya diversos modos de dar los contenidos para hacerlos atractivos y los jóvenes los perciben:

"Historia me gusta mucho porque la profe la da re lindo la hace re entretenida, creo que también influye mucho como te dan una materia para que te guste o no..." (A, sexo masculino, 20 años).

Otro joven afirmó: 
"Lo que más me gusta es que en filosofía por ejemplo hacemos un libro de ensayos y podemos escribir del tema que nosotros queremos, eso está bueno y a fin de año lo presentamos el año pasado hicimos uno y ahora estamos preparando otro" ( $\mathrm{N}$, sexo masculino, 18 años).

"La profe la da re lindo", "hace la materia re entretenida", "hacemos un libro de ensayos", "eso está bueno" son expresiones que muestran que los jóvenes reconocen estrategias implementadas por los profesores para motivarlos, sin embargo las mismas no logran revertir su percepción de aburrimiento. ¿Por qué? Corea y Lewkowicz (2004) plantean que la estrategia pedagógica de la motivación se ensayó con creces en la escuela y dio pobres resultados. Los autores remarcan que tal vez la falla no es la estrategia sino el modo de abordar el problema. En el mismo sentido Sibilia (2012) en un profundo análisis de la dinámica escolar en estos tiempos afirma que los cuerpos y las subjetividades para los cuales la escuela fue creada en el momento de su invención y durante su gradual solidificación, han cambiado y entran en tensión con los modos de ser y estar en el mundo que surgen hoy en día. La autora plantea que los jóvenes presentan otras subjetividades en otros cuerpos que ya no responden al disciplinamiento, la instrucción y la civilidad que impartía la Escuela Moderna. Son cuerpos en constante movimiento y transformación en un contexto informático, digital, interactivo y cambiante). La autora afirma además que:

El resultado suele ser el aburrimiento, la indiferencia o la frustración (...) el valor simbólico que envolvía al conocimiento con su poma de cultura letrada se ha desvanecido dejando en su lugar esos salones ahuecados en los cuales se producen innumerables desencuentros y fracasos, a veces sin embargo, en el mejor de los casos obligan a unos y otros inventar formas alternativas e innovadoras de habitar esa situación para que tenga algún sentido (Sibilia, 2012: 77).

El aburrimiento de los jóvenes entrevistados sería entonces una manifestación de este desfasaje, de esta transformación de cuerpos y subjetividades que habitan en un contexto escolar que conserva una identidad inmutable, inconmovible a través de las historias y las geografías.

Existe una incongruencia explica Lewkowicz entre lo que los jóvenes son y aquello que las instituciones esperan de los niños y jóvenes contemporáneos. "Por su conformación misma la institución no puede más que suponer el tipo subjetivo que la va a habitar pero actualmente la lógica social no entrega esa materia humana en las condiciones supuestas por la institución" (Corea y Lewkowicz, 2004: 106). En este punto se produce el desacople y el consecuente aburrimiento de los jóvenes. El motivo es muy simple, "porque su subjetividad se ha construido 
en la experiencia cotidiana mucho más mediática y mercantil de la contemporaneidad (...) su subjetividad pedagógica no ha sido convenientemente preformateada" (Sibilia, 2012: 76).

Pero son los mismos jóvenes los que expresan las soluciones para combatir el "aburrimiento":

Creo que deberíamos elegir las materias que queremos cursar, sí tener lengua, matemáticas y no sé si ética... esas sí obligatorias pero elegir otras materias que nos gustan para poder estudiar, más que nada para que después te sirvan con lo que vas a seguir ( $T$, sexo femenino, 20 años).

La propuesta de la joven de "elegir las materias" para que después "te sirvan con lo que vas a seguir", al igual que en otro testimonio que la joven reclama que "no creía que las materias fueran a servirle para el futuro" demanda una respuesta a la percepción de falta de utilidad de los conocimientos proporcionados en la escuela. Este es un tema de intensos debates en el ámbito educativo aunque cabe replantearse ¿qué lugar ocupan los jóvenes en esos debates?

\section{Experiencias escolares con las nuevas tecnologías}

Otra de las causas del aburrimiento y este desfasaje actual entre jóvenes y escuela parece ser este desacople entre el ritmo de vida instantáneo e inmediato que ofrecen las nuevas tecnologías y una gramática escolar fragmentada, estructurada, propia de otra época. Por lo tanto, el análisis de las experiencias de los jóvenes con las nuevas tecnologías en el contexto escolar permite analizar el modo en que estas interpelan a la escuela y el modo en el que re definen los sentidos que los jóvenes le otorgan al estudio.

Entre las principales modificaciones que introducen el uso de las nuevas tecnologías pueden mencionarse los cambios en el espacio y tiempo escolar ya que, como se mencionó anteriormente, internet ha generado nuevos lugares y momentos interactivos para propiciar el encuentro con el otro. El tiempo y espacio acelerado a un ritmo desmedido en la red es muy diferente a la estructura de la gramática escolar con tiempos pautados para cada materia y la separación estructurada entre mementos de estudio y tiempos de distracción con los diversos espacios destinados a cada momento.

Las redes atraviesan las experiencias escolares ya que funcionan todo el tiempo y en cualquier lugar, y tal como plantea Carlón (2012) reconfiguran las formas en que lo público, lo privado y lo íntimo se constituyeron e interrelacionaron en la modernidad. Inmersa en esta realidad la escuela incorpora el uso de la red social facebook como una herramienta en su cotidianeidad escolar 
Los jóvenes refirieron a sus discursos al uso de la página de facebook de la escuela:

"La escuela tiene una página de facebook y creo que eso es muy útil porque te enterás de un montón de cosas, te avisan cosas por ahí entonces está bueno" (A, sexo masculino, 20 años).

"La página de facebook de la escuela la usamos muchísimo, además es Angie -se refiere a la Directoraquien la administra así que los padres también están informadas las cosas por ahí o le hacen preguntas a ella por ese medio" ( $A$, sexo femenino, 17 años).

La página de facebook de la escuela es destacada por los jóvenes principalmente por la utilidad que les brinda como medio de información. La legitimidad de esta vía de comunicación tal como la destaca el discurso de la joven radica en que es la Directora del establecimiento quien la administra, hecho que agrega una cuota de legitimidad a la información que por allí se transmite. Esto implica resaltar que aunque los medios digitales han impulsado el desarrollo de redes horizontales de comunicación interactiva las relaciones de poder institucionalizadas no se diluyen sino que se legitiman en el campo de la comunicación y las nuevas tecnologías.

Respecto al uso de celulares dentro de la institución, los alumnos resaltaron su utilización con fines educativos aunque la normativa escolar prohíbe su uso dentro de la institución.

"Acá en el colegio lo usamos cuando nos dejan los profes. En realidad no se puede traer pero si pedís permiso te dejan, hay materias que nos dan tareas que es buscar en internet entonces ahí si los usamos" (A, sexo femenino, 17 años).

"Si en realidad no se puede traer pero como todos los traen, hay profes que lo usan para que busquemos cosas, por ejemplo uno de los profes de filosofía nos hace buscar información, para eso el profe tiene que activar el internet porque no está activo siempre para que no nos distraigamos" (A, sexo masculino, 20 años).

Es interesante detenerse en este aspecto. El celular es el medio de comunicación más usado por los jóvenes, sin embargo en la escuela su uso está prohibido.

En este sentido podría afirmarse que existe una concepción compartida entre los jóvenes y los docentes de la escuela que el celular interrumpe el normal funcionamiento del aula. Sin embargo los jóvenes destacan de esta institución en particular la relación de diálogo que existe entre alumnos-docentes y principalmente piensan que este clima se logra porque la escuela no les impone las reglas sino que éstas son consensuadas, una de esas reglas consensuadas es el uso del celular en clase más allá que la norma escolar lo prohíba. Estos sentidos también aparecieron en sus discursos al referirse al uso del celular en el aula "todos lo traen entonces 
los profes los usan para que busquemos cosas". Es decir, existen momentos de la jornada escolar en el que alumnos y docentes acuerdan el uso del teléfono celular en la clase.

El sentido con el que se usa en el contexto escolar principalmente es como una herramienta de búsqueda de información y de trabajo en clase. Si bien su uso es consensuado entre docentes y alumnos, son los profesores quienes regulan su uso dentro del aula y deciden cuando comienza el uso del celular y cuando termina. Situación a partir de la cual puede pensarse que aunque esta escuela en particular se caracteriza por no imponer reglas a sus alumnos, la relación no es horizontal sino que existe una relación de poder asimétrica dada por la autoridad del docente de la escuela y esa autoridad es legitimada por los estudiantes.

\section{¿Enseñanza presencial o educación virtual?}

Pensar el sentido de Internet en la escuela primero pasa por pensar el sentido mismo de la escuela. Por ello, otro aspecto importante para conocer el rol de las nuevas tecnologías en los sentidos juveniles otorgados a la escuela consistió en conocer si los jóvenes consideran que la estructura escolar podría ser reemplazada por la educación virtual. Las respuestas se organizaron en la siguiente figura: 


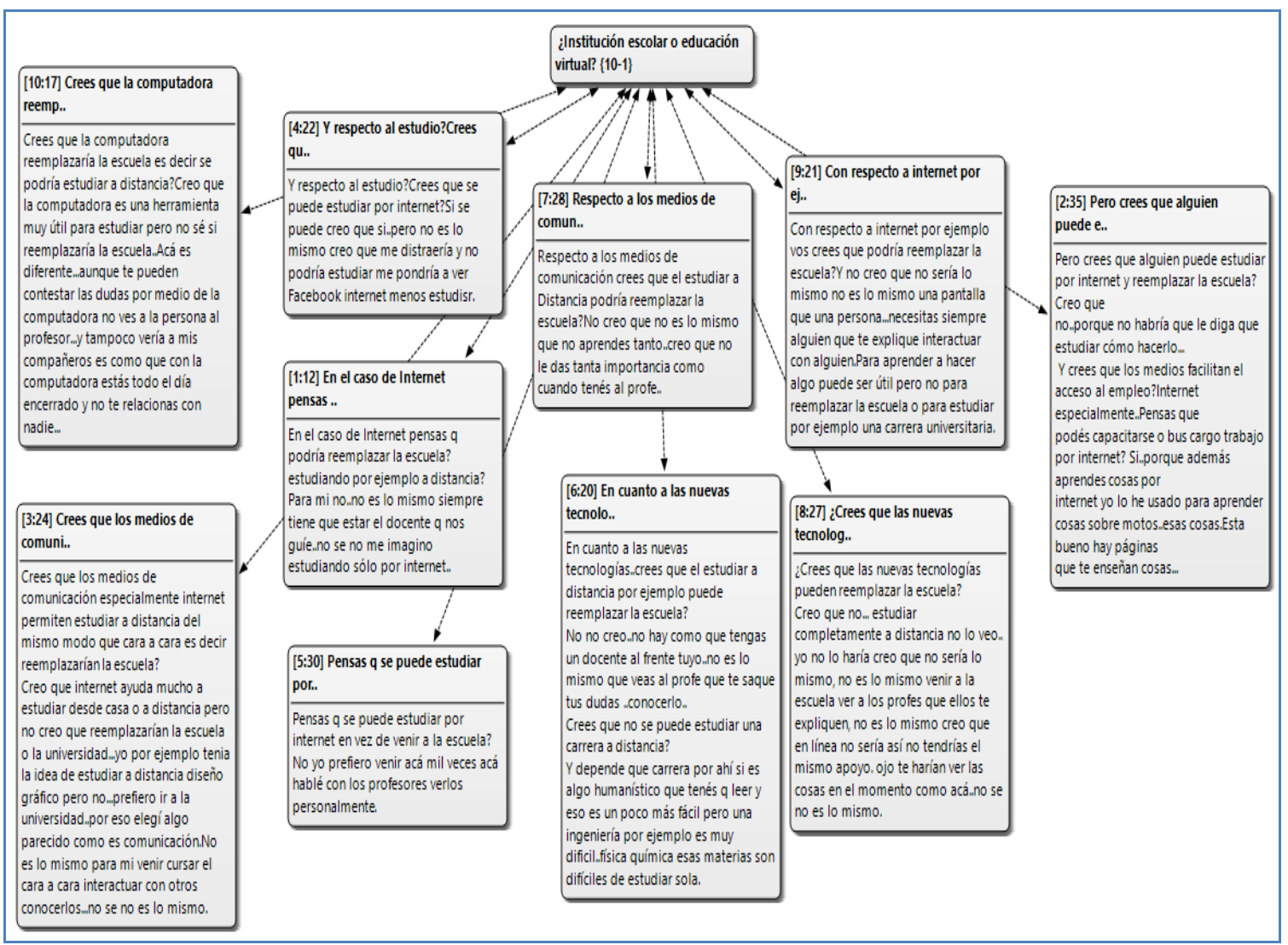

Figura 2. Sentidos juveniles de la institución escolar frente a la educación virtual.

La figura precedente está integrada por un solo código ¿Institución escolar o educación virtual? En las citas que lo componen puede leerse que la totalidad de los entrevistados respondieron que aunque se puede estudiar por internet no es una alternativa que ellos elegirían y que no sería lo mismo que asistir a la escuela.

"Si se puede creo que sí, pero no es lo mismo creo que me distraería y no podría estudiar me pondría a ver Facebook, internet, menos estudiar" ( $F$, sexo masculino, 17 años).

Internet ofrece un flujo de información constante, yuxtapuesta y continúa que parecería propiciar la dispersión y por lo tanto aunque la mencionan como herramienta de utilidad para la búsqueda de información, no así como reemplazo del estudio en la escuela. Por otra parte, tal como puede leerse en el gráfico precedente, el factor quizás más importante, ya que la mayoría hizo hincapié en ello, por el cual los jóvenes eligen la escuela presencial antes que la educación virtual es la necesidad del encuentro interpersonal. 
"No no creo, no hay como que tengas un docente al frente tuyo. No es lo mismo que veas al profe que te saque tus dudas conocerlo" (B, sexo femenino, 18 años).

"Creo que la computadora es una herramienta muy útil para estudiar pero no sé si reemplazaría la escuela. Acá es diferente, aunque te pueden contestar las dudas por medio de la computadora no ves a la persona, al profesor y tampoco vería a mis compañeros es como que con la computadora estás todo el día encerrado y no te relacionas con nadie ( $R$, sexo femenino, 17 años).

Las expresiones resaltadas en las afirmaciones de los jóvenes "no hay como que tengas un docente al frente tuyo", "por medio de la computadora no ves a la persona", "no vería al profesor", "tampoco a mis compañeros "con la computadora estás todo el día encerrado y no te relacionas con nadie"; refiere al valor del encuentro con un otro asequible a partir de todos los sentidos ,es decir se trata de un encuentro en el que el otro se hace visible y directamente accesible a la experiencia sensorial y perceptiva, característica que solo se posibilita en un espacio y tiempo compartido.

\section{A modo de reflexión final}

En este análisis se intentó aportar algunas herramientas para comprender los sentidos que los jóvenes (re)construyen sobre la escuela y la manera en que problematizan la creciente incompatibilidad entre los modos de ser y estar en el mundo actuales con la ya anticuada gramática escolar. A partir de los testimonios juveniles, puede considerarse que uno de los sentidos que otorgan a la escuela es el de "aburrimiento", principalmente porque en esta época de constantes estímulos provenientes del entorno, la motivación juega un papel muy importante en sus percepciones y el aburrimiento se presenta como un síntoma de los límites de la experiencia pedagógica para motivar a los alumnos. Aunque las nuevas tecnologías son utilizadas en la escuela como herramientas de aprendizaje siempre con un uso sugerido y regulado por el profesor, la gramática escolar no ha sufrido cambios estructurales que permitan a los jóvenes considerar a la escuela valiosa en tanto los conocimientos que proporciona. Sin embargo los jóvenes, al hacer alusión a su experiencia escolar particular -que transcurre en la escuela Leonardo Da Vinci de la ciudad de Villa Mercedes, San Luis, Argentina- refirieron a una institución a la que les gusta asistir, en la que construyen relaciones con sus pares y con los profesores y la directora desde el diálogo, la comprensión, la ayuda. Esta escuela no es valorada tanto por el proyecto educativo que ofrece ni por su modalidad de autogestión sino por los vínculos que en ella se construyen. Los jóvenes usaron los calificativo "contención" y 
"libertad" para referirse a la relación que tienen con los adultos de la escuela y destacaron el esfuerzo que hacen los profesores para transformar las actividades en atractivas e interesantes. De este modo puede afirmarse que los sentidos otorgados al estudio se encuentran impregnados por una valorización de la experiencia particular en esta institución en relación al afecto que les brinda. Frente al imaginario social que se escucha a diario que refiere a "el fin de la escuela", y contrariamente a los discursos que refieren a la crisis de la institución escolar, los jóvenes otorgan importancia a su trayectoria escolar, así sus experiencias escolares continúan ocupando un lugar importante en sus trayectorias de vida. Haciendo un analogismo con lo planteado por Corea y Lewkowicz (2004) cuando expresan que para pensar la infancia, hay que des-suponer la infancia y postular que hay chicos. De modo semejante puede afirmarse que para pensar los jóvenes hay que des-suponer lo que ellos necesitan y enfocarse en sus percepciones no pensarlos como los hombres del mañana sino como jóvenes de hoy y en función a ello colocarlos como protagonistas en el aquí y ahora.

\section{Bibliografía}

Bauman, Z. (2000). Modernidad líquida. Buenos Aires: Fondo de Cultura Económica.

Bergé, S. (2007). Gestión de personas en organizaciones públicas (Segunda edición). Rev. Caxias do Sul: Educs.

Bourdieu, P. (1988). Espacio social y poder simbólico. Revista de Occidente, 81, pp. 97-119.

Bourdieu, P. (1990). Sociología y cultura. México DF: Grijalbo.

Carlón, M. y Nieto, F. (2012). La política de los internautas. Nuevas formas de participación. Buenos Aires: La Crujía.

Castells, M. (2008). Comunicación, poder y contrapoder en la sociedad en red. Revista Telos: Cuadernos de comunicación e innovación, 75, pp. 11-23.

Cicioni, A. (1998). El movimiento de las charter schools. Una amenaza y una oportunidad para la educación pública argentina. Documentos de Trabajo. Fundación Gobierno y Sociedad. Recuperado de https://bit.ly/2R8TXwE

Chaves, M. (2005). Investigaciones sobre juventudes en Argentina: estado del arte en ciencias sociales (1983-2006). La Plata, Ciudad de Buenos Aires. Recuperado de https://bit.ly/2Vvdx4L

Chaves, M. (2010). Jóvenes, territorios y complicidades. Una antropología de la juventud urbana. Buenos Aires: Espacio Editorial. 
Corea, C. y Lewcowicz, I. (2004). Pedagogía del aburrido: Escuelas destituidas, familias perplejas. Buenos Aires: Paidós.

Duschatzky, S. (1999). La escuela como frontera. Reflexiones sobre la experiencia escolar de sectores populares. Buenos Aires: Paidós.

Feixa, C. (1999). De culturas, subculturas y estilos. En De jóvenes, bandas y tribus (pp. 84105). Barcelona: Ariel. Recuperado de https://bit.ly/2Augkly

Glaser, B. y Strauss, A. (1967). El muestreo teórico. En The discovery of grounded theory: strategies for qualitative research (pp. 45-77). New York: Aldine Publishing Company.

Larrosa, J. (1996). La experiencia de la lectura. Estudios sobre literatura y formación. Barcelona: Laertes.

Macri, M. (2010). Estudiar y trabajar: perspectivas y estrategias de los adolescentes. Buenos Aires, Argentina: La Crujía.

Margulis, M. y Urresti, M. (1996). La juventud es más que una palabra. En Margulis, M. (ed.). La juventud es más que una palabra. Buenos Aires: Biblos.

Núñez, P. y Litichever, L. (2015). Radiografías de la experiencia escolar .Ser joven(es) en la escuela. Ciudad Autónoma de Buenos Aires, Argentina: Grupo Editor UniversitarioClacso.

Sibilia, P. (2012). ¿Redes o paredes? La escuela en tiempos de dispersión. Buenos Aires, Argentina: Tinta Fresca.

Tiramonti, G. (2011). Variaciones sobre la forma escolar. Límites y posibilidades de la escuela media. Rosario, Argentina: FLACSO y Homo Sapiens. 